The team also pinpointed the roles of four proteins that form the protein envelope and that allow cells to stick to each other and to surfaces.

Science 337, 236-239 (2012)

\section{ASTRONOMY}

\section{Dark galaxies revealed}

'Dark' galaxies contain no stars, making them impossible to observe using optical telescopes. Now, Sebastiano Cantalupo and his colleagues at the University of California, Santa Cruz, have managed to detect a faint fluorescent glow from a few such galaxies.

The researchers used the European Southern Observatory's Very Large Telescope to look at bright galaxies known as quasars, which can illuminate nearby dark galaxies. The team was able to see the glowing outline of a dozen candidate dark galaxies, thanks to radiation from a quasar exciting the hydrogen gas in these galaxies.

The team estimates the dark galaxies contain a gas mass around one billion times that of the Sun. These galaxies could serve as reservoirs of hydrogen fuel for star formation in larger galaxies, the authors suggest. Mon. Not. R. Astron. Soc. http:// dx.doi.org/10.1111/j.13652966.2012.21529.x (2012)

\section{COGNITIVE NEUROSCIENCE}

\section{A smart hub in the brain}

Human intelligence could result from high levels of activity in a region of the brain called the lateral prefrontal cortex, as well as strong connectivity between this region and the rest of the brain.

Human intelligence is marked by the ability to direct thoughts and behaviour in pursuit of a goal. This 'cognitive control' has previously been linked to activity in the lateral prefrontal cortex. Using functional magnetic resonance imaging, Michael Cole at Washington University in Saint
Louis, Missouri, and his team examined the activity of this region and its connectivity across the brain in 94 young adults. Individuals with high levels of connectivity were more likely to perform well on tests of both cognitive control and intelligence.

The lateral prefrontal cortex could act as a global hub for human intelligence that exerts its effects across the brain.

J. Neurosci. 32, 8988-8999 (2012) \section{BIOPHYSICS \\ Trout nose yields magnetic cells}

Certain animals, including some birds and fish, are guided by magnetic fields, and researchers have isolated magnetic cells that could be at the root of this internal compass.

Michael Winklhofer at Ludwig-MaximiliansUniversity in Munich, Germany, and his colleagues took epithelial cells from the rainbow trout nose and exposed them under a microscope to a moderately strong, rotating magnetic field. A few of the cells spun at the same frequency as the magnetic field, indicating that they were sensitive to the field, whereas the other cells did not alter their behaviour. Highresolution imaging showed that the responsive cells had micrometre-scale structures composed of iron-rich crystals attached to their cell membranes. These caused the cells to align with the magnetic field.

Proc. Natl Acad. Sci. USA

http://dx.doi.org/10.1073/

pnas.1205653109 (2012)

\section{BIODIVERSITY \\ Extinctions still to come}

Up to $90 \%$ of extinctions due to Amazon rainforest loss have yet to occur, a modelling study suggests.

Robert Ewers and his team at Imperial College London in Ascot used deforestation

COMMUNITY CHOICE

\title{
p53 can be cancer's friend, not foe
}

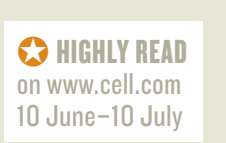

The protein p53 is well known for its role as a tumour suppressor; however, p53 can also help breast tumour cells to dodge the effects of chemotherapy.

Normal p53 activates programmed cell death, or apoptosis, and the p 53 gene is often mutated in cancer. Researchers expected that tumours with mutant p53 would be more difficult to treat than those with normal p53, but previous studies on breast cancer have not found this. Guillermina Lozano at the MD Anderson Cancer Center in Houston, Texas, and her team therefore tested the effects of a chemotherapy drug, doxorubicin, on mice bearing breast tumours with either normal or mutated $\mathrm{p} 53$.

Tumours in mice with normal p53 shrank little in response to treatment and relapsed more quickly than those with mutated p53. After treatment, many tumour cells with normal p53 entered a state of senescence in which they stopped dividing - however, the cells secreted signalling proteins that could trigger the proliferation of neighbouring cells, leading to relapse.

Cancer Cell 21, 793-806 (2012)

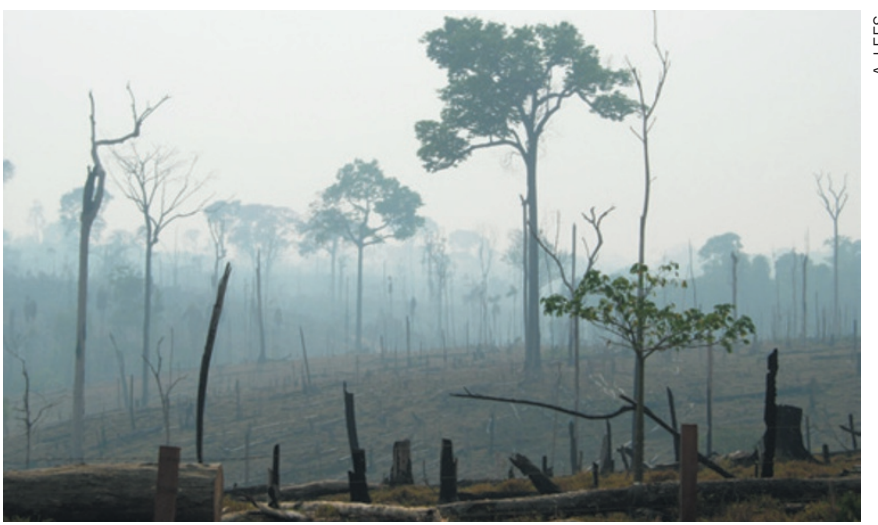

data from 1978 to 2008 - plus data on forest-dependent vertebrates - to create a model that relates species extinction to the timing and amount of habitat loss. The model takes into account the fact that species do not become extinct immediately after losing their habitats and that deforestation happens intermittently, rather than all at once as previous models have assumed. The authors calculated the number of species headed for extinction owing to previous deforestation, or the 'extinction debt': an average of two mammals, four or five birds, and one amphibian for every 2,500 square kilometres.

The team then projected Amazon extinctions up to 2050 on the basis of four scenarios of different levels of forest regulation. They found that in the most likely scenario, $60-70 \%$ of expected extinctions would be yet to come as a result of past and future habitat loss.

Science 337, 228-232 (2012)

For a longer story on this research, see http://go.nature.com/rmo2d3

\section{$\rightarrow$ NATURE.COM}

For the latest research published by Naturevisit:

www.nature.com/latestresearch 